\title{
Attractor bifurcation for FKPP type equation with periodic boundary condition
}

\author{
Qiang Zhang ${ }^{1,2}$
}

Correspondence: zqcs007@163.com ${ }^{1}$ College of Mathematics, Sichuan University, Chengdu, Sichuan 610064, P. R. China

Full list of author information is available at the end of the article

\begin{abstract}
In this article, we make bifurcation analysis on the FKPP type equation under periodic boundary condition. And we show that the solutions bifurcate from the trivial solution $u=0$ to an attractor $\Sigma_{\lambda}$ as parameter crosses certain critical value. Moreover, we prove that the attractor $\Sigma_{\lambda}$ consists of only one cycle of steady state solutions and is homeomorphic to $S^{1}$. The analysis is based on a new theory of bifurcation, called attractor bifurcation, which was developed by Ma and Wang. 2000 Mathematics Subject Classification: 35B; 35Q; 37G; 37L.
\end{abstract}

Keywords: FKPP type equation, periodic boundary condition, attractor bifurcation, center manifold

\section{Introduction}

We consider the following reaction diffusion equation of FKPP type:

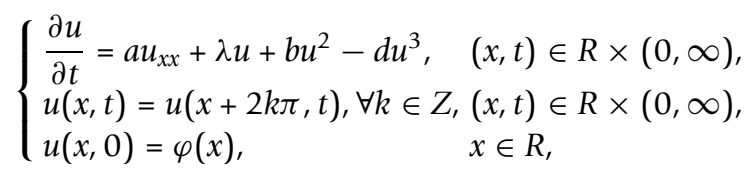

supplemented with the following natural constraint:

$$
\int_{0}^{2 \pi} u(x) d x=0
$$

where $a>0, d>0, b$ are given and $\lambda>0$ is system parameter.

In 1937, the FKPP equation was first proposed by Fisher as a model to describe the propagation of advantageous genes [1] and was studied mathematically by Kolmogorov et al. [2]. Moreover, it was also used as biological models for population dynamics [3-5].

The FKPP equation has been extensively studied during the last decades. Among all the topics of these, the existence of traveling waves (exact form of solutions) and the asymptotic behavior of solutions attract much attention. Many different kinds of methods for the existence of traveling waves (exact form of solutions) haven been developed, such as Painleve expansion method [6,7], bilinear method [8,9], symmetry methods [10]. On the other hand, many results on the asymptotic behavior of solutions are also obtained; see among others [11-14] and references therein.

(c) 2012 Zhang; licensee Springer. This is an Open Access article distributed under the terms of the Creative Commons Attribution License (http://creativecommons.org/licenses/by/2.0), which permits unrestricted use, distribution, and reproduction in any medium, provided the original work is properly cited. 
However, there is few work on the attractor bifurcation of the FKPP equation. As a new notion of bifurcation, attractor bifurcation was developed by Ma and Wang [15-17] and attracted researchers [18,19]. Ma and Wang [15] first proposed this new notion and applied it to Rayleigh-Benard Convection. Afterwards, with this new theory, Park [18] analyzed the bifurcation of the complex Ginzburg-Landau equation (CGLE) and Zhang et al. [19] studied the attractor bifurcation of the Kuramoto-Sivashinsky equation.

In this article, we focus on the attractor bifurcation of FKPP type Equation (1.1). The bifurcation analysis near the first eigenvalue of (1.1) will be discussed. The topology structure of the bifurcated solutions will also be studied. As a result, we show the system bifurcates from the trivial solution to an attractor $\Sigma_{\lambda}$ as system parameter $\lambda$ crosses the critical value $a$, the first eigenvalue of the eigenvalue problem of the linearized equation of (1.1). Furthermore, we prove that $\Sigma_{\lambda}$ is homeomorphic to $S^{1}$ and consists of only one cycle of steady state solutions.

This article is organized as follows. The mathematical setting are given in Section 2. The main results are stated in Section 3. The preliminaries are put in section 4. And Section 5 devote to the proof of the main theorem.

\section{Mathematical setting}

Let

$$
\begin{aligned}
H & =L^{2}(0,2 \pi), \\
H_{1} & =\left\{u \in H^{2}(0,2 \pi) \mid \int_{0}^{2 \pi} u(x) d x=0, u(x+2 k \pi)=u(x)\right\},
\end{aligned}
$$

and we define $L_{\lambda}=-A+B_{\lambda}: H_{1} \rightarrow H$ and $G: H_{1} \rightarrow H$ by

$$
\left\{\begin{array}{l}
A u=-a u_{x x}, \\
B_{\lambda} u=\lambda u, \\
G u=b u^{2}-d u^{3} .
\end{array}\right.
$$

Consequently, we have an operator equation which is equivalent to the problem (1.1):

$$
\left\{\begin{array}{l}
\frac{d u}{d t}=L_{\lambda} u+G(u) \\
u(x, 0)=\varphi
\end{array}\right.
$$

Thanks to the existence result of semi-linear evolution equations, see Temam [20], Pazy [21], we can define a semigroup

$$
S(t): H_{1} \rightarrow H, t \geq 0,
$$

which satisfies the semigroup property.

\section{Main results}

\subsection{The definition of attractor bifurcation}

In order to state the main theorem of this article, we start with the definition of attractor bifurcation which was first proposed by Ma and Wang in [15-17]. 
Let $H$ and $H_{1}$ be two Hilbert spaces, and $H_{1} \otimes H$ be a dense and compact inclusion. We consider the following nonlinear evolution equations

$$
\left\{\begin{array}{l}
\frac{d u}{d t}=L_{\lambda} u+G(u) \\
u(x, 0)=u_{0}
\end{array}\right.
$$

where $u:[0, \infty) \rightarrow H$ is the unknown function, $\lambda \in R$ is the system parameter, and $L_{\lambda}: H_{1}: \rightarrow H$ are parameterized linear completely continuous fields depending continuously on $\lambda$, which satisfy

$$
\left\{\begin{array}{l}
L_{\lambda}=-A+B_{\lambda} \quad \text { a sectorial operator, } \\
A: H_{1} \rightarrow H \text { a linear homeomorphism, } \\
B_{\lambda}: H_{1} \rightarrow H \text { a parameterized linear compact operators. }
\end{array}\right.
$$

Since $L_{\lambda}$ is a sectorial operator which generates an analytic semigroup $S_{\lambda}(t)=\left\{e^{t L_{\lambda}}\right\}_{t \geq 0}$ for any $\lambda \in R$, we can define fractional power operators $\left(-L_{\lambda}\right)^{\mu}$ for $0 \leq$ $\mu \leq 1$ with domain $H_{\mu}=D\left(\left(-L_{\lambda}\right)^{\mu}\right)$ such that $H_{\mu_{1}} \subset H_{\mu_{2}}$ if $\mu_{1}>\mu_{2}$, and $H_{0}=H$. In addition, we assume that the nonlinear terms $G(., \lambda): H_{\alpha} \rightarrow H$ for some $0 \leq \alpha<1$ are a family of parameterized $C^{r}$ bounded operator $(r \geq 1)$ continuously depending on $\lambda$, such that

$$
G(u, \lambda)=o\left(\|u\|_{H_{\alpha}}\right), \quad \forall \lambda \in R .
$$

Definition 3.1.1 A set $\Sigma \subset H$ is called an invariant set of (3.1) if $S(t) \Sigma=\Sigma$ for any $t$ $\geq 0$. An invariant set $\Sigma \subset H$ of (3.1) is said to be an attractor if $\Sigma$ is compact, and there exists a neighborhood of $W \subset H$ of $\Sigma$ such that for any $\psi_{0} \in W$ we have

$$
\lim _{t \rightarrow \infty} \operatorname{dist}_{H}\left(\psi\left(t, \psi_{0}\right), \Sigma\right)=0 .
$$

Definition 3.1.2 (1) We say that the solution to Equation (3.1) bifurcates from $(\psi, \lambda)$ $=\left(0, \lambda_{0}\right)$ to an invariant set $\Omega_{\lambda}$, if there exists a sequence of invariant sets $\left\{\Omega_{\lambda_{n}}\right\}$ of (3.1) such that $0 \notin \Omega_{\lambda_{n}}$, and

$$
\begin{gathered}
\lim _{n \rightarrow \infty} \lambda_{n}=\lambda_{0}, \\
\lim _{n \rightarrow \infty} \max _{x \in \Omega_{\lambda_{n}}}|x|=0 .
\end{gathered}
$$

(2) If the invariant sets $\Omega_{\lambda}$ are attractors of (3.1), then the bifurcation is called attractor bifurcation.

\subsection{Main theorem}

In this article, based on attractor bifurcation theory we obtain the following results.

Theorem 3.2.1 For the problem (1.1) with (1.2), if $2 b^{2}<9 a d$, following assertions hold true:

(1) if $\lambda \leq a$, the steady state $u=0$ is locally asymptotically stable. Furthermore, if $b=$ 0 , the steady state $u=0$ is globally asymptotically stable.

(2) if $\lambda>a$, the Equation (1.1) bifurcates from $u=0$ to an attractor $\Sigma_{\lambda}$ which is homeomorphic to $S^{1}$.

(3) $\Sigma_{\lambda}$ consists of exactly one cycle of steady solutions of (1.1).

(4) There exists a neighborhood $U$ of $u=0$, such that $\Sigma_{\lambda}$ attracts $U / \Gamma$, where $\Gamma$ is the stable manifold of $u=0$ with co-dimension 2 in $H$. 


\section{Preliminaries}

\subsection{Attractor bifurcation theory}

In the following, we proceed with the principle of exchange of stabilities (PES). Let the eigenvalues (counting multiplicity) of $L_{\lambda}$ be given by

$$
\beta_{k}(\lambda) \in C(k \geq 1) .
$$

Suppose that

$$
\begin{aligned}
& \Re \beta_{i}(\lambda)\left\{\begin{array}{l}
<0, \text { if } \lambda<\lambda_{0} \\
=0, \text { if } \lambda=\lambda_{0}(1 \leq i \leq m) \\
>0, \text { if } \lambda<\lambda_{0}
\end{array}\right. \\
& \Re \beta_{j}\left(\lambda_{0}\right)<0, \forall j \geq m+1 .
\end{aligned}
$$

Let the eigenspace of $L_{\lambda}$ at $\lambda=\lambda_{0}$ be

$$
E_{0}=\bigcup_{1 \leq j \leq m} \bigcup_{k=1}^{\infty}\left\{u, v \in H_{1} \mid\left(L_{\lambda_{0}}-\beta_{j}\left(\lambda_{0}\right)\right)^{k} w=0, w=u+i v\right\} .
$$

It is known that $\operatorname{dim} E_{0}=m$.

Theorem 4.1.1[17] Assume that the conditions (3.2), (3.3), (4.1), and (4.2) hold true, and $u=0$ is locally asymptotically stable for (3.1) at $\lambda=\lambda_{0}$. Then the following assertions hold true:

(1) (3.1) bifurcates from $(u, \lambda)=\left(0, \lambda_{0}\right)$ to attractors $\Omega_{\lambda}$, having the same homology as $S^{m-1}$, for $\lambda>\lambda_{0}$, with $m-1 \leq \operatorname{dim} \Omega_{\lambda} \leq m$, which is connected as $m>1$;

(2) for any $u_{\lambda} \in \Omega_{\lambda}, u_{\lambda}$ can be expressed as

$$
u_{\lambda}=v_{\lambda}+o\left(\left\|v_{\lambda}\right\|_{H_{1}}\right), v_{\lambda} \in E_{0} ;
$$

(3) There is an open set $U \subset H$ with $0 \in U$ such that the attractor $\Omega_{\lambda}$ bifurcated from $\left(0, \lambda_{0}\right)$ attracts $U / \Gamma$ in $H$, where $\Gamma$ is the stable manifold of $u=0$ with codimension $m$.

To get the structure of the bifurcated solutions, we introduce another theorem. Let $v$ be a two-dimensional $C^{r}(r \geq 1)$ vector field given by

$$
v_{\lambda}(x)=\lambda x-F(x, \lambda),
$$

for $x \in R^{2}$. Here

$$
F(x, \lambda)=F_{k}(x, \lambda)+o\left(|x|^{k}\right),
$$

where $F_{k}$ is a $k$-multilinear field, which satisfies an inequality

$$
C_{1}|x|^{k+1} \leq<F_{k}(x, \lambda), x>\leq C_{2}|x|^{k+1},
$$

for some constants $0<C_{1}<C_{2}$ and $k=2 m+1, m \geq 1$. 
Theorem 4.1.2 ([[16], Theorem 5.10, p. 134]) Under conditions (4.4) and (4.5), the vector field (4.3) bifurcates from $(x, \lambda)=(0,0)$ to an attractor $\Pi_{\lambda}$ for $\lambda>0$, which is homeomorphic to $S^{1}$. Moreover, one and only one of the following is true.

(1) $\Pi_{\lambda}$ is a period orbit,

(2) $\Pi_{\lambda}$ consists of infinitely many singular points,

(3) $\Pi_{\lambda}$ contains at most $2(k+1)=4(m+1)$ singular points, and has $4 N+n(N+n$ $\geq 1$ ) singular points, $2 N$ of which are saddle points, $2 N$ of which are stable node points (possibly degenerate), and $n$ of which have index zero.

\subsection{Center manifold reduction}

Since the key point in the proof of Theorem 3.2.1 is the center manifold function, we introduce an approximation formula of the center manifold function derived in [16].

We assume that the spaces $H_{1}$ and $H$ can be decomposed into

$$
\left\{\begin{array}{l}
H_{1}=E_{1}^{\lambda} \oplus E_{2}^{\lambda}, \operatorname{dim} E_{1}^{\lambda}<\infty, \text { near } \lambda_{0} \in R, \\
H=\tilde{E}_{1}^{\lambda} \oplus \tilde{E}_{2}^{\lambda}, \tilde{E}_{1}^{\lambda}=E_{1}^{\lambda}, \tilde{E}_{2}^{\lambda}=\text { the closure of } E_{2}^{\lambda} \text { in } H,
\end{array}\right.
$$

where $E_{1}^{\lambda}, E_{2}^{\lambda}$ are invariant spaces of $L_{\lambda}$, i.e., $L_{\lambda}$ can be decomposed into $L_{\lambda}=\mathcal{L}_{1}^{\lambda} \oplus \mathcal{L}_{2}^{\lambda}$ such that for any $\lambda$ near $\lambda_{0}$,

$$
\begin{aligned}
& \mathcal{L}_{1}^{\lambda}=\mathcal{L}_{\lambda} \mid E_{1}: E_{1}^{\lambda} \rightarrow \tilde{E}_{1}^{\lambda}, \\
& \mathcal{L}_{2}^{\lambda}=\mathcal{L}_{\lambda} \mid E_{2}: E_{2}^{\lambda} \rightarrow \tilde{E}_{2}^{\lambda},
\end{aligned}
$$

where all eigenvalues of $\mathcal{L}_{2}^{\lambda}$ possess negative real parts, all eigenvalues of $\mathcal{L}_{1}^{\lambda}$ possess nonnegative real parts at $\lambda=\lambda_{0}$.

Thus, for $\lambda$ near $\lambda_{0}$, (3.1) can be rewritten as

$$
\left\{\begin{array}{l}
\frac{d x}{d t}=\mathcal{L}_{1}^{\lambda}+G_{1}(x+y, \lambda), \\
\frac{d y}{d t}=\mathcal{L}_{2}^{\lambda}+G_{2}(x+y, \lambda),
\end{array}\right.
$$

where $u=x+y \in H_{1}, \quad x \in E_{1}^{\lambda}, \quad y \in E_{2}^{\lambda}, \quad G_{i}=P_{i} G(u, \lambda)$, and $P_{i}: H \rightarrow \tilde{E}_{i}$ are canonical projections. Moreover, let

$$
E_{2}^{\lambda}(\alpha)=\text { closure of } E_{2}^{\lambda} \text { in } H_{\alpha \prime}
$$

where $\alpha<1$ given by (3.3).

By the classical center manifold theorem (see among others [20,22]), there exists a neighborhood of $\lambda_{0}$, a neighborhood $B_{\lambda} \subset E_{1}^{\lambda}$ of $x=0$, and a $C^{1}$ center manifold function $\Phi(., \lambda): B_{\lambda} \rightarrow E_{2}^{\lambda}(\alpha)$, depending continuously on $\lambda$. Then to investigate the dynamic bifurcation of (3.1) it suffices to consider the finite dimensional system as follows

$$
\frac{d x}{d t}=\mathcal{L}_{1}^{\lambda}+G_{1}\left(x+\Phi_{\lambda}(x), \lambda\right), x \in B_{\lambda} \subset E_{1}^{\lambda} .
$$

Assume the nonlinear operator $G$ be in the following form

$$
G(u, \lambda)=G_{k}(u, \lambda)+o\left(\|u\|^{k}\right),
$$


for some integer $k \geq 2$. Here $G_{k}$ is a k-multilinear operator

$$
\begin{gathered}
G_{k}: H_{1} \times \cdots \times H_{1} \rightarrow H, \\
G_{k}(u, \lambda)=G_{k}(u, \cdots, u, \lambda) .
\end{gathered}
$$

Theorem 4.2.1 ([[16], Theorem 3.8, p. 63]) Under the conditions (3.2), (4.1), (4.2), (4.6), and (4.8), the center manifold function $\Phi(x, \lambda)$ can be expressed as

$$
\Phi(x, \lambda)=\left(-\mathcal{L}_{2}^{\lambda}\right)^{-1} P_{2} G_{k}(x, \lambda)+O\left(|\Re \beta|\|x\|^{k}\right)+o\left(\|x\|^{k}\right),
$$

where $\mathcal{L}_{2}^{\lambda}$ is as in (4.7), $P_{2}: H \rightarrow \tilde{E}_{2}$ the canonical projection, $x \in E_{1}^{\lambda}$, and $\beta=\left(\beta_{1}^{\lambda}, \ldots, \beta_{m}^{\lambda}\right)$ the eigenvalues of $\mathcal{L}_{1}^{\lambda}$.

\section{Proof of main theorem}

In this section, we shall prove Theorem 3.2.1 by four steps.

Step 1. In this step, we shall study the eigenvalue problem of the linearized equation of (2.1) and shall find the eigenvectors and the critical value of $\lambda$.

Consider the eigenvalue problem of the linear equation,

$$
L_{\lambda} u=\beta u \text {. }
$$

Note that the eigenvalues $\left\{\rho_{k}\right\}_{k=1}^{\infty}$ and eigenvectors $\left\{e_{k}\right\}_{k=1}^{\infty}$ of Laplace operator, which satisfy

$$
\left\{\begin{array}{l}
\frac{d^{2}}{d x^{2}} e_{k}=\rho_{k} e_{k} \\
e_{k}(x+2 k \pi)=e_{k}(x) \\
\int_{0}^{2 \pi} e_{k}(x) d x=0 \\
\int_{0}^{2 \pi} e_{k}^{2}(x) d x=1
\end{array}\right.
$$

are

$$
\left\{\begin{array}{l}
\rho_{k}=-k^{2}, \\
e_{k}=\frac{\sin \mathrm{kx}}{\sqrt{\pi}} \quad \text { or } \quad \frac{\cos \mathrm{kx}}{\sqrt{\pi}} .
\end{array}\right.
$$

Then the eigenvalues and eigenvectors of (5.1) are

$$
\left\{\begin{array}{l}
\beta_{2 k-1}=\beta_{2 k}=\lambda-a k^{2} \\
e_{2 k-1}=\frac{\sin \mathrm{kx}}{\sqrt{\pi}}, e_{2 k}=\frac{\cos \mathrm{kx}}{\sqrt{\pi}} .
\end{array}\right.
$$

Now, we get the PES:

$$
\begin{gathered}
\beta_{1}(\lambda)=\beta_{2}(\lambda)\left\{\begin{array}{l}
<0, \lambda<a \\
=0, \lambda=a \\
>0, \lambda>a
\end{array}\right. \\
\beta_{j}(a)<0, j \geq 3 .
\end{gathered}
$$

As a result, conditions (4.1) and (4.2) are verified.

Step 2. We verify that for any $\lambda \in R$, operator $L_{\lambda}+G$ satisfies conditions (3.2) and (3.3).

From the theory of elliptic equations, operator $A: H_{1} \rightarrow H$ is a homeomorphism. Note that conclusion $H_{1} \otimes H$ is compact, then operator $B_{\lambda}: H_{1} \rightarrow H$ is linear compact 
operator. Thanks to the results of analytic semigroup in [20-22], from (5.2) we know that operator $L_{\lambda}: H_{1} \rightarrow H$ is a sectorial operator which generates an analytical semigroup. Condition (3.2) is verified.

It is easy to get the following inequality

$$
\begin{aligned}
\|G(u)\|_{H}^{2} & =\int_{0}^{2 \pi}\left|b u^{2}-d u^{3}\right|^{2} d x \\
& \leq C \int_{0}^{2 \pi}\left(u^{4}+u^{6}\right) d x \\
& \leq C\left(\|u\|_{L^{4}(0,2 \pi)}^{4}+\|u\|_{L^{6}(0,2 \pi)}^{6}\right) \\
& \leq C\left(\|u\|_{H_{1}}^{4}+\|u\|_{H}^{6} \frac{1}{2}\right),
\end{aligned}
$$

which implies that $G(u)=o\left(\|u\|_{H_{1}} \frac{1}{2}\right)$, where

$$
H_{\frac{1}{2}}=\left\{u \in H^{1}(0,2 \pi) \mid \int_{0}^{2 \pi} u(x) d x=0, u(x+2 k \pi)=u(x)\right\},
$$

condition (3.3) is verified.

Step 3. In this part, we shall prove the existence of attractor bifurcation and analyze the topological structure of attractor $\Sigma_{\lambda}$.

Let $E_{1}^{\lambda}=E_{0}=\operatorname{span}\left\{e_{1}, e_{2}\right\}, E_{2}^{\lambda}=E_{0}^{\perp}$. Let $\Phi$ be the center manifold function, in the neighborhood of $(u, \lambda)=(0, a)$, we have

$$
u=y+\Phi(\gamma)
$$

where $y=x_{1} e_{1}+x_{2} e_{2}$.

Then the reduction equations of (2.1) are as follows

$$
\left\{\begin{array}{l}
\frac{d x_{1}}{d t}=(\lambda-a) x_{1}+<G(u), e_{1}> \\
\frac{d x_{2}}{d t}=(\lambda-a) x_{2}+<G(u), e_{2}>.
\end{array}\right.
$$

To get the exact form of the reduction equations, we need to obtain the expression of $<G(u), e_{1}>$ and $<G(u), e_{2}>$.

Let $G_{2}: H_{1} \times H_{1} \rightarrow H$ and $G_{3}: H_{1} \times H_{1} \times H_{1} \rightarrow H$ are the bilinear and trilinear operators of $G$, respectively, i.e.,

$$
\begin{gathered}
G_{2}\left(u_{1}, u_{2}\right)=b u_{1} u_{2}, \\
G_{3}\left(u_{1}, u_{2}, u_{3}\right)=-d u_{1} u_{2} u_{3} .
\end{gathered}
$$

Since

$$
<G_{2}\left(x_{1} e_{1}+x_{2} e_{2}, x_{1} e_{1}+x_{2} e_{2}\right), e_{1}>=<G_{2}\left(x_{1} e_{1}+x_{2} e_{2}, x_{1} e_{1}+x_{2} e_{2}\right), e_{2}>=0,
$$


the first-order approximation of (5.3) doesn't work. Now, we shall find out the second-order approximation of (5.3). And the most important of all is to obtain the approximation expression of the center manifold function.

Since

$$
\begin{aligned}
& <G_{2}\left(x_{1} e_{1}+x_{2} e_{2}, x_{1} e_{1}+x_{2} e_{2}\right), e k> \\
& =b x_{1}^{2} \int_{0}^{2 \pi} e_{1}^{2} e_{k} d x+b x_{2}^{2} \int_{0}^{2 \pi} e_{2}^{2} e_{k} d x+2 b x_{1} x_{2} \int_{0}^{2 \pi} e_{1} e_{2} e_{k} d x
\end{aligned}
$$

and

$$
\begin{aligned}
& \int_{0}^{2 \pi} e_{1}^{2} e_{k} d x=0, \quad k \neq 4, \int_{0}^{2 \pi} e_{1}^{2} e_{4} d x=-\frac{1}{2 \sqrt{\pi}}, \\
& \int_{0}^{2 \pi} e_{2}^{2} e_{k} d x=0, \quad k \neq 4, \int_{0}^{2 \pi} e_{2}^{2} e_{4} d x=\frac{1}{2 \sqrt{\pi}}, \\
& \int_{0}^{2 \pi} e_{1} e_{2} e_{k} d x=0, \quad k \neq 3, \int_{0}^{2 \pi} e_{1} e_{2} e_{3} d x=\frac{1}{2 \sqrt{\pi}},
\end{aligned}
$$

we can obtain

$$
<G_{2}\left(x_{1} e_{1}+x_{2} e_{2}, x_{1} e_{1}+x_{2} e_{2}\right), e_{k}>=\left\{\begin{array}{l}
\frac{b}{\sqrt{\pi}} x_{1} x_{2}, k=3, \\
\frac{b}{2 \sqrt{\pi}} x_{2}^{2}-\frac{b}{2 \sqrt{\pi}} x_{1}^{2}, k=4, \\
0, k \neq 3,4 .
\end{array}\right.
$$

By the formula (4.9) in Theorem 4.2.1 and (5.7), the center manifold function $\Phi$, in the neighborhood of $(u, \lambda)=(0, a)$, can be expressed as

$$
\begin{aligned}
\Phi(y) & =-\sum_{k=3}^{\infty} \beta_{k}^{-1}<G_{2}(y, y), e_{k}>e_{k}+O\left(\left(\left|\beta_{1}\right|^{2}+\left|\beta_{2}\right|\right)^{2}|y|^{2}\right)+o\left(|\gamma|^{2}\right) \\
& =-(\lambda-4 a)^{-1} \frac{b}{2 \sqrt{\pi}}\left(2 x_{1} x_{2} e_{3}-x_{2}^{2} e_{4}+x_{1}^{2} e_{4}\right) \\
& +O\left(|\lambda-a|^{2}\left(\left|x_{1}\right|^{2}+\left|x_{2}\right|^{2}\right)+o\left|x_{1}\right|^{2}+\left|x_{2}\right|^{2}\right) .
\end{aligned}
$$

In the following, we calculate $<G(u), e_{j}>, j=1,2$.

$$
\begin{aligned}
<G(u), e_{j}> & =<G_{2}(\gamma+\Phi(\gamma), y+\Phi(\gamma)), e_{j}>+<G_{3}(\gamma+\Phi(\gamma), y+\Phi(\gamma), y+\Phi(y)), e_{j}> \\
& =<G_{2}(y, \Phi(\gamma)), e_{j}>+<G_{2}(\Phi(\gamma), y), e_{j}>+G_{3}(\gamma, y, y), e_{j}> \\
& +O\left(|\lambda-a|^{2}\left(\left|x_{1}\right|^{3}+\left|x_{2}\right|^{3}\right)+o\left(\left|x_{1}\right|^{3}+\left|x_{2}\right|^{3}\right), \quad j=1,2 .\right.
\end{aligned}
$$

Next we only need to find out $<G_{2}(y, \Phi(y)), e_{j}>,<G_{2}(\Phi(y), y), e_{j}>$ and $G_{3}(y, y, y), e_{j}$ $>$. 
By calculation, we have

$$
\begin{aligned}
& <G_{2}(\gamma, \Phi(\gamma)), e_{1}>=<G_{2}(\Phi(\gamma), \gamma), e_{1}> \\
& =\int_{0}^{2 \pi} b y \Phi(\gamma) e_{1} d x \\
& =-(\lambda-4 a)^{-1} \frac{b_{2}}{2 \sqrt{\pi}} \int_{0}^{2 \pi}\left(x_{1} e_{1}^{2}+x_{2} e_{1} e_{2}\right)\left(2 x_{1} x_{2} e_{3}-x_{2}^{2} e_{4}+x_{1}^{2} e_{4}\right) d x \\
& +O\left(|\lambda-a|^{2}\left(\left|x_{1}\right|^{3}+\left|x_{2}\right|^{3}\right)+o\left(\left|x_{1}\right|^{3}+\left|x_{2}\right|^{3}\right)\right. \\
& =-(\lambda-4 a)^{-1} \frac{b^{2}}{2 \sqrt{\pi}} \int_{0}^{2 \pi}\left(-x_{1}^{3} e_{1}^{2} e_{4}+x_{1} x_{2}^{2} e_{1}^{2} e_{4}+2 x_{1} x_{2}^{2} e_{1} e_{2} e_{3}\right) d x \\
& +O\left(|\lambda-a|^{2}\left(\left|x_{1}\right|^{3}+\left|x_{2}\right|^{3}\right)+o\left(\left|x_{1}\right|^{3}+\left|x_{2}\right|^{3}\right),\right. \\
& <G_{2}(\gamma, \Phi(\gamma)), e_{2}>=<G_{2}(\Phi(\gamma), \gamma), e_{2}> \\
& =\int_{0}^{2 \pi} b y \Phi(\gamma) e_{2} d x \\
& =-(\lambda-4 a)^{-1} \frac{b^{2}}{2 \sqrt{\pi}} \int_{0}^{2 \pi}\left(x_{2} e_{2}^{2}+x_{1} e_{1} e_{2}\right)\left(2 x_{1} x_{2} e_{3}-x_{2}^{2} e_{4}+x_{1}^{2} e_{4}\right) d x \\
& +O\left(|\lambda-a|^{2}\left(\left|x_{1}\right|^{3}+\left|x_{2}\right|^{3}\right)+o\left(\left|x_{1}\right|^{3}+\left|x_{2}\right|^{3}\right)\right. \\
& =-(\lambda-4 a)^{-1} \frac{b^{2}}{2 \sqrt{\pi}} \int_{0}^{2 \pi}\left(2 x_{1}^{2} x_{2} e_{1} e_{2} e_{3}-x_{1}^{2} x_{2} e_{2}^{2} e_{4}+x_{2}^{3} e_{2}^{2} e_{4}\right) d x \\
& +O\left(|\lambda-a|^{2}\left(\left|x_{1}\right|^{3}+\left|x_{2}\right|^{3}\right)+o\left(\left|x_{1}\right|^{3}+\left|x_{2}\right|^{3}\right)\right. \text {. }
\end{aligned}
$$

Note that (5.4) hold true, we have

$$
\begin{aligned}
&<G_{2}(y, \Phi(y)), e_{1}>=-(\lambda-4 a)^{-1} \frac{b^{2}}{4 \pi} x_{1}^{3}-(\lambda-4 a)^{-1} \frac{b^{2}}{4 \pi} x_{1} x_{2}^{2} \\
&+ O\left(|\lambda-a|^{2}\left(\left|x_{1}\right|^{3}+\left|x_{2}\right|^{3}\right)+o\left(\left|x_{1}\right|^{3}+\left|x_{2}\right|^{3}\right),\right. \\
&<G_{2}(\gamma, \Phi(y)), e_{2}>=-(\lambda-4 a)^{-1} \frac{b^{2}}{4 \pi} x_{2}^{3}-(\lambda-4 a)^{-1} \frac{b^{2}}{4 \pi} x_{1}^{2} x_{2} \\
&+O\left(|\lambda-a|^{2}\left(\left|x_{1}\right|^{3}+\left|x_{2}\right|^{3}\right)+o\left(\left|x_{1}\right|^{3}+\left|x_{2}\right|^{3}\right) .\right.
\end{aligned}
$$

Since

$$
\int_{0}^{2 \pi} e_{1}^{4} d x=\int_{0}^{2 \pi} e_{2}^{4} d x=\frac{3}{4 \pi}, \quad \int_{0}^{2 \pi} e_{1}^{2} e_{2}^{2} d x=\frac{1}{4 \pi},
$$

we have

$$
\begin{aligned}
& <G_{3}(y, y, y), e_{1}>=\int_{0}^{2 \pi}-d y^{3} e_{1} d x=-d \int_{0}^{2 \pi}\left(x_{1}^{3} e_{1}^{4}+3 x_{1} x_{2}^{2} e_{1}^{2} e_{2}^{2}\right) d x=-\frac{3 d}{4 \pi} x_{1}^{3}-\frac{3 d}{4 \pi} x_{1} x_{2}^{2}, \\
& <G_{3}(y, y, y), e_{2}>=\int_{0}^{2 \pi}-d y^{3} e_{2} d x=-d \int_{0}^{2 \pi}\left(3 x_{1}^{2} x_{2} e_{1}^{2} e_{2}^{2}+x_{2}^{3} e_{2}^{4}\right) d x=-\frac{3 d}{4 \pi} x_{2}^{3}-\frac{3 d}{4 \pi} x_{1}^{2} x_{2},
\end{aligned}
$$


then we obtain the expression of $\left\langle G(u), e_{j}>, j=1,2\right.$.

$$
\begin{aligned}
& <G(u), e_{1}>=A x_{1}^{3}+A x_{1} x_{2}^{2}+O\left(|\lambda-a|^{2}\left(\left|x_{1}\right|^{3}+\left|x_{2}\right|^{3}\right)+o\left(\left|x_{1}\right|^{3}+\left|x_{2}\right|^{3}\right),\right. \\
& <G(u), e_{2}>=A x_{1}^{2} x_{2}+A x_{2}^{3}+O\left(|\lambda-a|^{2}\left(\left|x_{1}\right|^{3}+\left|x_{2}\right|^{3}\right)+o\left(\left|x_{1}\right|^{3}+\left|x_{2}\right|^{3}\right),\right.
\end{aligned}
$$

where $A=-(\lambda-4 a)^{-1} \frac{b^{2}}{2 \pi}-\frac{3 d}{4 \pi}$.

Put (5.8) into (5.3), we have the approximation of reduction equation

$$
\left\{\begin{array}{l}
\frac{d x_{1}}{d t}=(\lambda-a) x_{1}+A x_{1}^{3}+A x_{1} x_{2}^{2}+O\left(|\lambda-a|^{2}\left(\left|x_{1}\right|^{3}+\left|x_{2}\right|^{3}\right)+o\left(\left|x_{1}\right|^{3}+\left|x_{2}\right|^{3}\right) ，\right. \\
\frac{d x_{2}}{d t}=(\lambda-a) x_{2}+A x_{1}^{2} x_{2}+A x_{2}^{3}+O\left(|\lambda-a|^{2}\left(\left|x_{1}\right|^{3}+\left|x_{2}\right|^{3}\right)+o\left(\left|x_{1}\right|^{3}+\left|x_{2}\right|^{3}\right) .\right.
\end{array}\right.
$$

For the case of $\lambda<a$, it is obviously that $u=0$ is locally asymptotically stable. For the case of $\lambda=a$, if $9 a d>2 b^{2}$, which implies that $A<0$, then $u=0$ is also locally asymptotically stable. In particular, if $b=0, \lambda \leq a$, by Poincaré inequality we have

$$
\begin{aligned}
\frac{1}{2} \frac{d}{d t}\|u\|_{H}^{2} & =-a\left\|u_{x}\right\|_{H}^{2}+\lambda\|u\|_{H}^{2}-d\|u\|_{H}^{4} \\
& \leq(\lambda-a)\left\|u_{x}\right\|_{H}^{2}-d\|u\|_{H}^{4} \\
& \leq-d\|u\|_{H}^{4},
\end{aligned}
$$

which implies that $u=0$ is globally asymptotically stable. Assertion (1) of Theorem 3.2.1 be proved.

Since the following equality holds true

$$
x_{1}\left(A x_{1}^{3}+A x_{1} x_{2}^{2}\right)+x_{2}\left(A x_{1}^{2} x_{2}+A x_{2}^{3}\right)=A\left(x_{1}^{2}+x_{2}^{2}\right)^{2},
$$

according to Theorem 4.1.2, we can conclude that if $\lambda>a$, the equation (1.1) bifurcates from $u=0$ to an attractor $\Sigma_{\lambda}$ which is homeomorphic to $S^{1}$. Assertion (2) and (4) of Theorem 3.2.1 are proved.

Step 4. In the last step, we shall show that the bifurcated attractor of (2.1) contains a singularity cycle.

By Krasnoselskii Theorem for potential operator, at least, $L_{\lambda}+G$ bifurcates from $(u$, $\lambda)=(0, a)$ to a steady solutions $\left(V_{\lambda}, \lambda\right)$.

Since the solutions of (2.1) are translation invariant,

$$
V_{\lambda}(x, t) \rightarrow V_{\lambda}(x+\theta, t), \quad \forall \theta \in R,
$$

the set

$$
T=\left\{V_{\lambda}(x+\theta, t) \mid \theta \in R\right\}
$$

represents $S^{1}$ in $H_{1}$, which implies that assertion (3) of Theorem 3.2.1 is proved.

\section{Acknowledgements}

The author was very grateful to the anonymous referees whose careful reading of the manuscript and valuable comments enhanced presentation of the manuscript. This research was supported by Scientific Research Foundation of Civil Aviation Flight University of China (J2011-30). 
The author declares that he has no competing interests.

Received: 31 December 2011 Accepted: 13 April 2012 Published: 13 April 2012

\section{References}

1. Fisher, RA: The wave of advance of advantageous genes. Ann Eugenics. 7, 353-369 (1937)

2. Kolmogorov, A, Petrovskii, I, Piskunov, N: Étude de l'équation de la diffusion avec croissance de la quantité de lamatieré et son application á un probléme biologique. Moscow Univ Bull Math. 1, 1-25 (1937)

3. Aronson, DG, Weinberger, HF: Nonlinear diffusion in population genetics, combustion, and nerve propagation. Partial Differential Equations and Related Topics. In Lecture Notes in Mathematics, vol. 446,Springer-Verlag, New York (1975)

4. Fife, PC: Mathematical aspects of reacting and diffusing systems. In Lecture Notes in Biomathematics, vol. 28,SpringerVerlag, Berlin/New York (1979)

5. Murray, JD: Mathematical Biology. Springer-Verlag, New York (1989)

6. Harrison, BK, Estabrook, FB: Geometric approach to invariance groups and solution of partial differential systems. J Math Phys. 12, 653-666 (1971). doi:10.1063/1.1665631

7. Ablowitz, MJ, Zeppetella, A: Explicit solutions of Fisher's equation for a special wave speed. Bull Math Biol. 41(6), $835-840$ (1979)

8. Kawahaka, T, Tanaka, M: Interactions of traveling fronts: an exact solution of a nonlinear diffusion equation. Phys Lett A. 97(8), 311-314 (1983). doi:10.1016/0375-9601(83)90648-5

9. Ma, WX, Fuchssteiner, B: Explicit and exact solutions to a Kolmogorov-Petrovskii-Piskunov equation. Int J non-Liear Mech. 31(3), 329-338 (1996). doi:10.1016/0020-7462(95)00064-X

10. Clarkson, PA, Mansfield, EL: Symmetry reductions and exact solutions of a class of nonlinear heat equations. Phys D. 70(3), 250-288 (1994). doi:10.1016/0167-2789(94)90017-5

11. Kametaka, Y: On the nonlinear diffusion equation of Kolmogorov-Petrovskii-Piskunov type. Osaka J Math. 13, 11-66 (1976)

12. Hamel, F, Roques, L: Fast propagation for KPP equations with slowly decaying initial conditions. J Diff Equ. 249, 1726-1745 (2010). doi:10.1016/j.jde.2010.06.025

13. Moet, HJK: A note on the asymptotic behavior of solutions of the KPP equation. SIAM J Math Anal. 10(4), 728-732 (1979). doi:10.1137/0510067

14. Rodrigo, M, Mimura, M: Annihilation dynamics in the KPP-Fisher equation. Eur J Appl Math. 13, 195-204 (2002)

15. Ma, T, Wang, SH: Atttactor bifurcation theory and its applications to Rayleigh-Bénard convection. Commun. Pure Appl Anal. 2(4), 591-599 (2003)

16. Ma, T, Wang, SH: Bifurcation Theory and Applications. In Nonlinear Science Series A, vol. 53,World Scientific, Singapore (2005)

17. Ma, T, Wang, SH: Dynamic bifurcation of nonlinear evolution equations and applications. Chinese Annals Math. 26(2), 185-206 (2005). doi:10.1142/S0252959905000166

18. Park, J: Bifurcation and stability of the generalized complex Ginzburg-Landau equation. Communications on Pure and Applied Analysis. 7(5), 1237-1253 (2008)

19. Zhang, YD, Song, LY, Axia, W: Dynamical bifurcation for the Kuramoto-Sivashinsky equation. Nonlinear Analysis: Theory, Methods and Applications. 74(4), 1155-1163 (2011). doi:10.1016/j.na.2010.09.052

20. Temam, R: Infinite-Dimensional Dynamical Systems in Mechanics and Physics. In Appl Math Sci, vol. 68,Springer, New York (1997)

21. Pazy, A: Semigroups of Linear Operators and Applications to Partial Differential Equations. In Appl Math Sci, vol. 44, Springer, New York (2006)

22. Henry, D: Geometric Theory of Semilinear Parobolic Equations. In Lectrue Notes in Matheatics, vol. 840,Springer-Verlag, New York (1982)

doi:10.1186/1687-2770-2012-41

Cite this article as: Zhang: Attractor bifurcation for FKPP type equation with periodic boundary condition. Boundary Value Problems 2012 2012:41.

\section{Submit your manuscript to a SpringerOpen ${ }^{\circ}$ journal and benefit from:}

- Convenient online submission

Rigorous peer review

- Immediate publication on acceptance

- Open access: articles freely available online

- High visibility within the field

- Retaining the copyright to your article

Submit your next manuscript at $\boldsymbol{\wedge}$ springeropen.com 